\title{
Adhesive Contact of Nanowire in Three-Point Bending Test
}

\author{
Yin Zhang and Ya-Pu Zhao* \\ State Key Laboratory of Nonlinear Mechanics, Institute of Mechanics, Chinese Academy of \\ Sciences, Beijing 100190, China
}

Received in final form 18 July 2009; revised 8 November 2009; accepted 8 December 2009

\begin{abstract}
A new adhesive receding contact model is presented in this paper for a nanowire in a three-point bending test. Because of its flexability, the nanowire in such a test, may lift-off or separate from its supporting elastic medium; this can dramatically change the nanowire boundary conditions and deformations. The changes of the nanowire boundary conditions and deformations, have a significant impact on the interpretation of the experimental data of the nanowire material properties. Through the model developed here, some explanations are offered for the different and contradicting observations of the nanowire material properties and the nanowire boundary conditions, found in recent experiments.

(C) Koninklijke Brill NV, Leiden, 2011
\end{abstract}

\section{Keywords}

Adhesion, nanowire, Young's modulus, receding contact, lift-off, boundary conditions

\section{Nomenclature}

$E_{1}, v_{1}$ Young's moduli and Poisson's ratios of nanowire, respectively.

$E_{2}, \nu_{2}$ Young's moduli and Poisson's ratios of elastic supporting medium, respectively.

$E^{*}$ Reduced modulus and $1 / E^{*}=\left(1-v_{1}^{2}\right) / E_{1}+\left(1-v_{2}^{2}\right) / E_{2}$.

$\gamma$ The surface energy per unit area of a surface and $2 \gamma$ is the work of adhesion.

$L_{\mathrm{S}}$ Suspension span.

$L_{1}$ and $L_{2}$ Lengths of nanowire portions laid on the trench support.

$R$ Nanowire radius.

\footnotetext{
* To whom correspondence should be addressed. Tel.: +86-10-8254 3932; Fax: +86-10-8254 3977; e-mail: yzhao@imech.ac.cn 
$I=\pi R^{4} / 4$ Nanowire cross-section moment of inertia.

$P_{\mathrm{e}}$ Line load $\left(\mathrm{N} \cdot \mathrm{m}^{-1}\right)$ due to nanowire contact with elastic medium.

$k_{1}=\pi E^{*} / 2$ Elastic foundation modulus.

$k_{2}=2 \sqrt[4]{2 R\left(\pi E^{*} \gamma\right)^{2}}$ Coefficient of nonlinear softening spring.

$W$ Nanowire deflection.

$F$ Concentrated vertical load.

$\delta_{\mathrm{D}}$ Dirac delta function.

$\beta=\sqrt[4]{\left(E^{*} /\left(2 E_{1}\right)\right)}(1 / R)$ Parameter (with unit of $\mathrm{m}^{-1}$ ) introduced to nondimensionalize the governing equation.

$l_{1}=\beta L_{1}, l_{2}=\beta L_{2}$ Dimensionless lengths of portion laid on elastic medium.

$l_{\mathrm{s}}=\beta L_{\mathrm{s}}$ Dimensionless suspension span.

$w=\beta W$ Dimensionless nanowire deflection.

$\alpha=\left(k_{2} / k_{1}\right) \beta^{3 / 4}$ A dimensionless parameter indicating the (order) of adhesion contribution to the line load as compared with that due to the Hertzian contact.

$J=F /\left(4 \beta^{2} E_{1} I\right)$ Dimensionless concentrated vertical load.

\section{Introduction}

The so-called one-dimensional (1D) nanostructures [1], such as micro/nanotubes [2-4], nanobelts [5] and nanowires [6-11] are important types of materials used in gas sensors, high-frequency resonators, nanoscale light-emitting diodes, highresolution tips for atomic force microscopes (AFM), scanning tunneling microscopes (STM), photovoltaics, etc. The mechanical properties of these 1D micro/nanomaterials are of interest for both technical and theoretical reasons. Due to its high spatial resolution and the accuracy/sensitivity of direct force measurements, the bending test with an AFM is commonly used for mechanical characterizations. The 1D micro/nanomaterials in these tests are first suspended over porous material [2-4, 6], or a strip [5], or an etched hole [7], or over a trench [8-11]. The AFM tip then exerts a concentrated force on the suspended section to form a typical three-point bending test [7]. The AFM tip is often placed at the center of the suspended section, which is thus referred to as the midpoint test [7, 8]. By assuming the Euler-Bernoulli beam model, [12] the midpoint displacement has the following relationship with the testing material properties:

$$
k=\frac{F}{z_{\mathrm{mp}}}=K \frac{E_{1} I}{L_{\mathrm{s}}^{3}},
$$


where $k$ is the effective spring stiffness of the beam and the AFM measures the data for the force-displacement $\left(F-z_{\mathrm{mp}}\right)$ curves $[5,7-11]$. The term $F$ is the concentrated load exerted at the center of the beam; $z_{\mathrm{mp}}$ is the beam midpoint displacement which is also the maximum displacement for a clamped-clamped $(\mathrm{C}-\mathrm{C})$ beam and a hinged-hinged $(\mathrm{H}-\mathrm{H})$ beam with the load at its center. $K$ is a constant depending on the testing materials boundary conditions; $K=192$ for the $\mathrm{C}-\mathrm{C}$ boundary conditions and $K=48$ for the $\mathrm{H}-\mathrm{H}$ boundary conditions. In equation (1), $L_{\mathrm{S}}$ is the beam suspension length; $E_{1}$ is the beam (bending) Young's modulus and $I$ is the beam cross-sectional moment of inertia and $I=\pi R^{4} / 4$ for a solid circular beam ( $R$ is the radius). Therefore, by measuring the midpoint displacement; the concentrated load; the suspension length; the radius; and choosing the boundary conditions accordingly, the Young's modulus of the testing material can be found from above equation. Keep in mind that the spring stiffness $k$ of a $\mathrm{C}-\mathrm{C}$ beam (with the same $E_{1}, R$ and $L_{\mathrm{s}}$ ) is four times larger than that of a $\mathrm{H}-\mathrm{H}$ beam because of the difference in the $K$ values.

It should be noticed that for the three-point bending test of a nanowire which is bonded to the support by adhesion, the two boundary conditions at the nanowire two ends can be either $\mathrm{C}-\mathrm{C}$ or $\mathrm{H}-\mathrm{H}[2,5,8,9]$.

Because of the small size of nanowire/nanotube (which results in the large surface to volume ratio), and because of the large adhesion effect [2-7], most researchers assume $\mathrm{C}-\mathrm{C}$ boundary conditions for a nanowire/belt/tube suspended on a trench/pore. From equation (1) the Young's modulus can be calculated from $E_{1}=k L_{\mathrm{s}}^{3} /(K I)$ with $k$ supplied by the $F-z_{\mathrm{mp}}$ data, measured by the AFM. With $K$ fixed at 192 for a C-C beam, $I(R), L_{\mathrm{S}}$ can be measured with relatively high accuracy. By analyzing the experimental $F-z_{\mathrm{mp}}$ data [2, 5-7], many researchers found that the Young's modulus of Ag nanowire increases significantly with a decrease of its diameter - usually 2-3 times that of the bulk value. Cuenot et al. [6] and Jing et $a l$. [7] offer a surface stress theory to explain the increase in the Young's modulus with decreasing Ag nanowire diameter. According to their theory, the increase of the Ag nanowire Young's modulus is proportional to $R^{-3}$, which is also supported by their experimental observations [6,7]. However, the experiment by $\mathrm{Wu}$ et al. [10] on Ag nanowires does not agree with the trend observed in previous experiments and theories [6,7]. Wu's experiment shows that most of the measured Ag nanowires Young's moduli are higher than the bulk one but their Young's moduli are not sensitive to the change of nanowire radius at all, as shown in their Fig. 3 [10]; a novel fivefold twin microstructure mechanism is proposed to explain the increase of the Ag nanowire Young's modulus [10]. Here it is worth mentioning that there is another possible mechanism responsible for the larger Young's modulus observation/calculation. The equation $E_{1}=k L_{\mathrm{s}}^{3} /(K I)$ derived from equation (1) does not consider the axial force effect. Now because the AFM tip-nanowire friction coefficient is high (due to the large adhesion effect), it induces an additional tensile stress inside the nanowire [13] and as a result the axial tension stiffens the structure [14, 15]. Therefore the tension and also Young's modulus increase can both contribute 
to the increase of $k\left(k=F / z_{\mathrm{mp}}\right)$, as measured in the experiment. In essence the equation $E_{1}=k L_{\mathrm{s}}^{3} /(K I)$ (or $k=K\left(E_{1} I / L_{\mathrm{s}}^{3}\right) L_{\mathrm{s}}$ ) takes into account the Young's modulus increase, to explain the increase of $k$. Unlike the midpoint test which measures the force-displacement data only at the suspension center [2-7, 10], Chen et $a l$. conducted the three-point bending test by measuring force-displacement data for the whole profile of the suspended portions of the testing nanowires $[8,9]$. The reason for conducting the multiple-point measurements rather than the single point measurement is that unlike the model used in those experiments $[2-7,10]$ which assumes the $\mathrm{C}-\mathrm{C}$ boundary conditions for the testing nanowires, Chen et al. found that the boundary conditions of the suspended nanowire may change with the change of the diameter/load. This was verified by their experimental data $[8,9]$. With multiple-point force-displacement data and curve fitting, the boundary conditions can be specified $[8,9]$. By taking into account that boundary conditions may change, Chen et al. conclude that in their Ag nanowire test the Young's modulus of Ag nanowire was not significantly different from the bulk property [8]. Now for the Young's modulus test of the Ag nanowires, three different and contradicting trends were observed: (1) Cuenot et al. [6] and Jing et al. [7] observed that the Young's moduli of Ag nanowires (diameter range of 30-250 nm in [6] and $20-140 \mathrm{~nm}$ in [7]) increases monotonously with the decrease of the diameter and they were all larger than the bulk property; (2) Wu et al. [10] observed that the Young's moduli of Ag nanowires (diameter range of 13-35 nm) can be either larger or smaller than the bulk property and Young's moduli were found not to be sensitive to the nanowires diameters at all; (3) Chen et al. concluded that there is no size effect for Ag nanowires (diameter range of 65-140 nm) [8]. We are fully aware that the $\mathrm{Ag}$ nanowires from different groups are fabricated/processed differently, which can significantly change the nanowire material properties. However, Chen $e t$ $a l$. $[8,9]$ pointed out that the single point measurements as those done in references $[2-7,10]$ may suffer from the drawback of assuming incorrect boundary conditions. The same contradicting experimental observations on other types of nanowire Young's modulus measurements were also noticed by Park and Klein [16]. They used the surface Cauchy-Born (SCB) model to compute the eigenfrequencies of a gold nanowire and they found that for the same gold nanowires, the eigenfrequencies of those with clamped-free $(\mathrm{C}-\mathrm{F})$ boundary conditions were reduced and the eigenfrequencies of those with the $\mathrm{C}-\mathrm{C}$ ones were increased [16], which is another way of saying that the effective Young's modulus of the $\mathrm{C}-\mathrm{F}$ gold nanowire became smaller and that of the $\mathrm{C}-\mathrm{C}$ became larger. Their SCB model shows that boundary conditions behave as a constraining condition which determines the eigenfrequencies of a nanowire together with its surface effect [16]. Again, in Park and Klein's model [16], the boundary conditions are set $a$ priori.

This paper presents a model describing the boundary conditions transition and deformations of nanowires suspended on a trench with their ends bonded and supported by adhesion. Chen et al. [8,9] came to the following conclusions in their experiments with both $\mathrm{Ag}$ and GaN nanowires: (1) the nanowire boundary condi- 
tions were found to be $\mathrm{C}-\mathrm{C}$ for those with small diameters and $\mathrm{H}-\mathrm{H}$ for those with larger diameters (in their experiments the suspended length $L_{\mathrm{S}}$ is fixed); (2) the magnitude of the applied concentrated force influenced on the boundary conditions transition; (3) a nanowire can have asymmetric boundary conditions of the clamped-hinged (C-H) type; (4) as shown in their Fig. 3(d) and 3(e) of reference [8], the deflection of a nanowire with a relatively large diameter is between a $\mathrm{C}-\mathrm{C}$ deflection curve and a $\mathrm{H}-\mathrm{H}$ curve. Chen et al. also noticed intermediate boundary conditions [9]. All these experimental observations can be explained by the model presented in this work. Chen et al. [9] believe that adhesion and its competition with the applied load is the key to understanding the boundary conditions transition and our model agrees with that. We do not rule out the possibility that the Young's modulus of nanowire can be different from the bulk one because the thermodynamically fewer imperfections, microstructures and surface effects of nanowires can all alter the nanowire material properties. In this paper we assume the Young's modulus of the nanowire is fixed and we change the parameters such as the nanowire suspended length, the length of nanowire portion laid down on the support, radius, concentrated load magnitude and adhesion to see how the nanowire deforms and their boundary conditions change. All those parameters, mentioned above, have significant impact on the nanowire boundary conditions. In the three-point bending test for nanowires bonded with support by adhesion, the boundary conditions are a key issue. As indicated in equation (1), the boundary conditions, being either $\mathrm{C}-\mathrm{C}$ or $\mathrm{H}-\mathrm{H}$, can contribute to a Young's modulus evaluation being four times the other value. In our model we find that the nanowire boundary conditions in general are the intermediate ones, which is to say that the nanowire end is neither clamped nor hinged. The hinged end can not take any bending moment and a rotation will occur; the clamped end can take a bending moment and there is no rotation. For the end with the intermediate boundary conditions, it can take some bending moment but there is also a rotation. The intermediate boundary conditions are common in many as-grown or as-deposited film/substrate structures [17]. The nanowire boundary conditions can be asymmetric, of the clamped-hinged $(\mathrm{C}-\mathrm{H})$ type $[8,9]$, with $K \approx 107$ in equation (1) for the $\mathrm{C}-\mathrm{H}$ beam in the midpoint test [12]. The effective structure stiffness of a beam with the intermediate boundary conditions or with the asymmetric $\mathrm{C}-\mathrm{H}$ boundary conditions, is between that with $\mathrm{H}-\mathrm{H}$ boundary conditions and that with $\mathrm{C}-\mathrm{C}$ boundary conditions. The fact is that a nanowire with intermediate boundary conditions or with $\mathrm{C}-\mathrm{H}$ type during three-point bending test may offer some insight into the experimental observations that the $\mathrm{Ag}$ nanowire Young's modulus is $2-3$ times larger than its bulk one $[6,7]$.

Even in those experiments which assume the $\mathrm{C}-\mathrm{C}$ boundary conditions, the researchers all show their concerns for the boundary conditions of nanowires under test and realize the importance of boundary conditions on determining the nanowire Young's modulus [2-7]. Sliding is one of the mechanisms which may cause the change of boundary conditions $[4,5,7,8]$. Although the AFM exerts a vertical force, the AFM tip-nanowire contact [13] and the mid-plane stretching during 


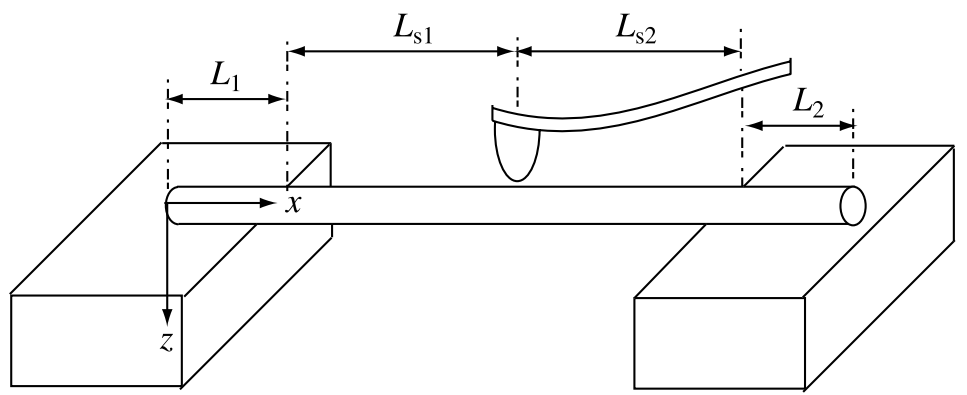

(a)

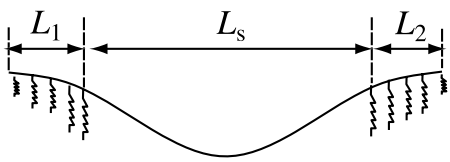

(I)

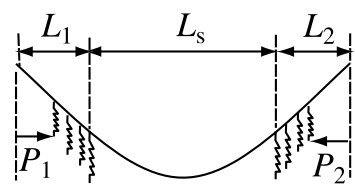

(III)

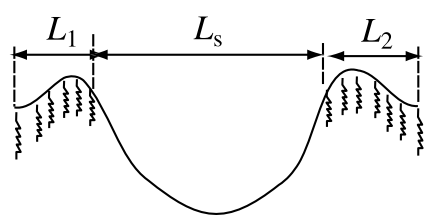

(II)

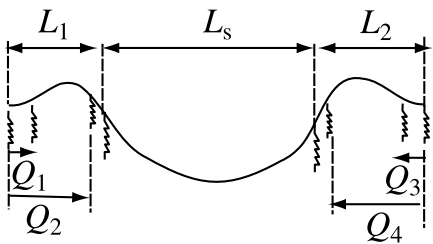

(IV)

(b)

Figure 1. (a) Dimension of a nanowire under an AFM loading and its coordination system. The schematic diagram of a beam laid on a trench. The beam dimensions and the coordinate system are also shown. (b) Four typical boundary conditions of the beam under loading: (I) and (II) full contact, (III) and (IV) partial contact.

nanowire deflection [18], can both induce a horizontal tensile force which can cause the nanowire in a test to slide. Here the vertical direction is the $z$-axis direction and horizontal direction is the $x$-axis direction as shown in Fig. 1(a). However, the experimental observations show very little or no sliding [4, 5, 7]. Lift-off was proposed as another possible mechanism causing the boundary conditions to change $[2,8]$. Unlike sliding which occurs in horizontal direction and can be observed relatively easily by SEM imaging [5, 7], lift-off occurs in a vertical direction, which is also experimentally difficult to observed in the three-point bending test of nanowires. Cuenot et al. [2] assumed that strong adhesion should prevent lift-off and thus the boundary conditions should be the $\mathrm{C}-\mathrm{C}$ type. However, Chen et al. [9] suspected that under a relatively large applied force, the adhesion may not be sufficient enough to hold the nanowire ends clamped. Paulo et al. pointed out that 'the nonideal anchoring using adhesion forces' will introduce 'interfacial mechanical instabilities', which means the nanobeam may not be 'solidly connected to prefabricated microstructures' [19]. To achieve 'a mechanically rigid anchor' (which is 
the clamped boundary condition), Paulo et al. used the vapor-liquid-solid (VLS) method to grow the nanowires from small catalyst particles deposited on a substrate [19]. Similarly, in order to make sure the nanowires two ends are clamped after they are dispersed on a trench, Zhu et al. [1] and Wu et al. [10] enhanced the nanowires bonding with the trench by electron-beam-induced deposition (EBID) welding. The adhesion force is a relatively weak force and its effect stands out only when the surface to volume ratio is large. When the nanowire diameter is small (the surface to volume ratio is large) and applied force is relatively small, the adhesion effect can be strong enough to hold the nanowire two ends clamped. However, when the nanowire diameter is large or the applied force is large, the adhesion may not be strong enough to hold the two ends clamped, which leads to the boundary condition transition from the $\mathrm{C}-\mathrm{C}$ type to the $\mathrm{H}-\mathrm{H}$ one.

Lift-off is an essential topic in the research of receding contact [20, 21], unbonded contact [22-24] and tensionless contact [25-27]. In the Hertzian contact of the flexural structures with an elastic medium [20-27], which has no adhesion, there is only compressive stress inside the contact area. The fact that the contacting flexural structures deflect and that the contacting bodies react differently to the tension and compression are the reasons for the lift-off; and lift-off reduces the contact area. The term 'receding contact' emphasizes the fact that the contact area is smaller when loaded; the name of unbonded contact emphasizes the fact that the structure is allowed to lift-off/separate from its contacting medium; the name of tensionless contact emphasizes the fact that the unilateral response of two contacting bodies, i.e., the contacting bodies react only in compression. Yu et al. [28] developed a model for the carbon nanotube contact under indentation using Hertz contact and ignoring the nanotube flexibility. The nanowires/nanobelts/nanotubes are the ones with large aspect ratio of length to radius/width, which results in the large flexibility. The contact of flexible structures is significantly different from $\mathrm{rigid} / \mathrm{stiff}$ ones. One of the significant differences is that the contact length of the (very) flexible structures is independent of the load magnitude because of lift-off [20-27], which is very counter-intuitive. It is worth pointing out that those models [20-26] do not specify how 'flexible' the structures are and assume there is no initial gap/separation between two contacting body; Zhang and Murphy [27] show that only when the structure is flexible enough and there is no gap, can the contact length of flexible structures be independent of load magnitude. For the chunky structure or for the case when two bodies are separated at the beginning, the contact length is dependent on the load magnitude [27]. For the highly flexible 1D nanostructures, the receding contact model is an appropriate one [29]. In the nanowire contact, adhesion is also an important issue as discussed above. Chaudhury et al. [30] and Chen et al. [31] developed an adhesive model for a cylinder contact with an elastic half-space, but the flexibility of cylinder was again ignored. Adhesion induces tensile force inside the contact area and as the result the adhesive contact (for example, Johnson-Kendall-Roberts (JKR) model) has a larger contact area and deeper normal displacement than the Hertzian one [32]. This paper models the contact of 
the nanowire with the trench support as an adhesive receding contact. In our model the adhesion is to prevent/reduce lift-off and thus has the significant impact on the boundary conditions transition as observed by Chen et al. [8,9]. The formulation of the receding contact problem can be either integral [20-25] or differential [26, 27]. If the supporting elastic medium is modeled as an elastic half-space, the contact problem is in essence to solve a Boussinesq problem [32] and its formulation can only be integral. When the supporting elastic medium is modeled on an elastic foundation, the formulation can be either differential or integral. From a modeling aspect, the elastic half-space model is a mathematically more difficult problem than the elastic foundation one [33]. Compared with the differential formulation, the integral formulation is rather complex, lengthy and mathematically challenging. This paper uses the elastic foundation model and has a differential formulation for the contact problem. However, the parameters of the elastic foundation are derived from the elastic half-space model.

\section{Model Development}

Figure 1(a) shows a cylinder with the Young's modulus $E_{1}$, Poisson's ratio $v_{1}$, radius $R$ laid down on a trench with the Young's modulus $E_{2}$ and Poisson's ratio $v_{2} . L_{1}$ and $L_{2}$ are the lengths of the cylinder portions laid on the trench support when there is no applied load. $L_{\mathrm{s} 1}$ and $L_{\mathrm{s} 2}$ are the lengths from the loading point to the left and right edges, respectively. $L_{\mathrm{s}}=L_{\mathrm{s} 1}+L_{\mathrm{s} 2}$ is thus the cylinder suspension length. The coordinate system is also shown in the Fig. 1(a). We mention above that the cylinder may lift-off from the trench support, which leads to the different contact scenarios. Figure 1(b) shows four different typical contact scenarios and these contact scenarios as discussed later in detail are the key to understand the cylinder deformation with different loading, adhesion and geometric conditions.

Let us first derive the pressure-displacement relation in the trench support area. When the elastic medium is modeled as an elastic half space and for the time being adhesion is ignored, the line load $P_{\mathrm{e}}$ resulted from the contact between the cylinder and trench support is given as follows [32]:

$$
P_{\mathrm{e}}=\frac{\pi a^{2} E^{*}}{4 R}
$$

Here $1 / E^{*}=\left(1-v_{1}^{2}\right) / E_{1}+\left(1-v_{2}^{2}\right) / E_{2}$. The following relation holds [32]:

$$
W=R-\sqrt{R^{2}-a^{2}} \approx \frac{a^{2}}{2 R},
$$

where $W$ is the displacement of the cylinder center sinking into the elastic medium [31] and $a$ is the half contact width. In the above equation the approximation is only valid when $a / R$ is very small [32]. So assuming a small $a / R$, we obtain the following equation in conjunction with equations (2) and (3):

$$
P_{\mathrm{e}}=\frac{\pi a^{2} E^{*}}{4 R}=\frac{\pi E^{*}}{2} W=k_{1} W .
$$


Here $k_{1}$ is the elastic foundation modulus defined as $k_{1}=\left(\pi E^{*}\right) / 2$. When the adhesion effect is considered, the following equation holds [30]:

$$
P_{\mathrm{e}}=\frac{\pi a^{2} E^{*}}{4 R}-\sqrt{4 E^{*} \pi \gamma a},
$$

where $\gamma$ is the surface energy per unit area of a surface (with the unit of $\mathrm{N} \cdot \mathrm{m}^{-1}$ ) [34], which is one half of the one defined in reference [30]. Again by applying the geometric relation of equation (3), equation (5) is now written as the following:

$$
P_{\mathrm{e}}=k_{1} W-k_{2} W^{1 / 4} \text {. }
$$

Here $k_{2}$ is defined as $k_{2}=2 \sqrt[4]{2 R\left(\pi E^{*} \gamma\right)^{2}}$. Clearly by considering the adhesion effect, the elastic foundation model of the elastic medium in this pressuredisplacement relation becomes nonlinear. Because here $-k_{2}<0$, the elastic foundation will behave as a softening spring [35]. When $k_{2}$ is zero, equation (6) recovers equation (4). The energy stored by the elastic foundation is as follows:

$$
\int_{C} \int_{0}^{W} P_{\mathrm{e}} \mathrm{d} W \mathrm{~d} x=\int_{C}\left(\frac{k_{1} W^{2}}{2}-\frac{4}{5} k_{2} W^{5 / 4}\right) \mathrm{d} x .
$$

Here $C$ is the contact domain of the cylinder, which is unknown and as shown later is dependent on various factors such as the applied force magnitude, nanowire geometry and adhesion etc. In Fig. 1(a), when the full contact scenario is seen in cases I and II, the domain $C$ consists of two parts $0 \leqslant x \leqslant L_{1}$ and $L_{1}+L_{\mathrm{s}} \leqslant$ $x \leqslant L_{1}+L_{\mathrm{s}}+L_{2}$. For the partial contact scenario, the domain $C$ can be rather complicate. For example, $C$ in case III is $\left[P_{1}, L_{1}\right] \cup\left[L_{1}+L_{\mathrm{s}}, L_{1}+L_{\mathrm{s}}+L_{2}-P_{2}\right]$; $C$ in case IV is $\left[0, Q_{1}\right] \cup\left[Q_{2}, L_{1}\right] \cup\left[L_{1}+L_{\mathrm{s}}, L_{1}+L_{\mathrm{s}}+L_{2}-Q_{4}\right] \cup\left[L_{1}+L_{\mathrm{s}}, L_{1}+\right.$ $\left.L_{\mathrm{s}}+L_{2}-Q_{3}\right]$.

We will discuss later how we determine those contact zones.

The system energy $E$ is now written as:

$$
\begin{aligned}
E= & \int_{0}^{L_{1}+L_{2}+L_{\mathrm{s}}} \frac{E_{1} I W_{x x}^{2}}{2} \mathrm{~d} x+\int_{C}\left(\frac{k_{1} W^{2}}{2}-\frac{4}{5} k_{2} W^{5 / 4}\right) \mathrm{d} x \\
& -\int_{0}^{L_{1}+L_{2}+L_{\mathrm{s}}} F \delta_{\mathrm{D}}\left(x-x_{\mathrm{o}}\right) W \mathrm{~d} x .
\end{aligned}
$$

Here $W_{x x}=\mathrm{d}^{2} W / \mathrm{d} x^{2}$ and $I$ is the second moment of inertia defined as $I=$ $\pi R^{4} / 4 ; \delta_{\mathrm{D}}$ is the Dirac delta function and $x_{\mathrm{o}}=L_{1}+L_{\mathrm{s} 1}$ is the loading point of the concentrated force $F$. The first term is the bending energy of the cylinder, the second one is the energy stored in the elastic foundation and the third is the work done by the concentrated load. If the principle of minimum potential energy (PMPE) is applied, i.e., $\delta E=0$, the following governing equation is obtained:

$$
\begin{cases}E I W_{x x x x}=F \delta_{\mathrm{D}}\left(x-x_{\mathrm{o}}\right), & \text { noncontact, } \\ E I W_{x x x x}+k_{1} W-k_{2} W^{-1 / 4}=0, & \text { contact. }\end{cases}
$$

The above governing equations of the beam for the contact and noncontact domain 
are similar to those in references [26, 27]. The solution approach used by Weitsman [26], Zhang and Murphy [27] is to give the analytical solution forms with the undetermined constants first and then solve those constants by using the matching/boundary conditions. There are two reasons why the solution approach used in $[26,27]$ cannot be used here. Firstly, when there is adhesion (i.e., $k_{2} \neq 0$ ), the governing equation in the contact domain is nonlinear and its analytical solution form cannot be obtained. Here it is worth mentioning that even when $k_{2}=0$, equation (9) as a whole is still nonlinear because of the unknown property of contact domain resulting from lift-off. Secondly, in the references [26, 27], the so-called 'continuous' contact scenario is assumed, which in essence prescribes the beam/cylinder deformation shape. The tensionless contact problem in references $[26,27]$ is that the whole beam is originally laid down on an elastic foundation and the assumption of the continuous contact scenario is proved to be valid in the elastic range [26]. Here our case is that only (small) portions of the cylinder around the two ends are originally laid down on the trench support, the contact scenario here is much more complicate. Figure 1(b) shows four typical scenarios and there are other types of contact. Especially in Fig. 1(b) case IV of partial contact scenario, the 'discontinuous' contact is formed: i.e., different contact zones are formed and separated from one another, which makes the above assumption of 'continuous' contact invalid in references [26, 27]. The variety and unknown property of the possible deformation shapes of the cylinder around the trench support make the solution approach used in references [26, 27] extremely difficult if not impossible to be applied here. Here the finite element approach is used to solve the problem. Before we start the finite element formulation of the problem, the following nondimensionalization scheme is introduced [26, 27]:

$$
\begin{aligned}
& \xi=\beta x, \quad \xi_{\mathrm{o}}=\beta x_{\mathrm{o}}, \quad w=\beta W, \quad l_{1}=\beta L_{1}, \quad l_{2}=\beta L_{2}, \\
& l_{\mathrm{s} 1}=\beta L_{\mathrm{s} 1}, \quad l_{\mathrm{s} 2}=\beta L_{\mathrm{s} 2}, \quad l_{\mathrm{s}}=\beta L_{\mathrm{s}}, \quad J=\frac{F}{4 \beta^{2} E_{1} I} .
\end{aligned}
$$

Here $\beta$ is defined as follow:

$$
\beta=\sqrt[4]{\frac{k_{1}}{4 E_{1} I}}=\sqrt[4]{\frac{E^{*}}{2 E_{1}}} \frac{1}{R},
$$

where $\beta$ has the unit of $\mathrm{m}^{-1}$. The PMPE is applied again and the following dimensionless equation is derived from equations (8) and (10):

$$
\begin{aligned}
& \int_{0}^{l_{1}+l_{2}+l_{s}} \frac{1}{4} w_{\xi \xi} \delta w_{\xi \xi} \mathrm{d} \xi+\int_{c}\left(w-\alpha w^{1 / 4}\right) \mathrm{d} x \\
& =\int_{0}^{l_{1}+l_{2}+l_{\mathrm{s}}} J \delta_{\mathrm{D}}\left(\xi-\xi_{\mathrm{o}}\right) \delta w \mathrm{~d} \xi
\end{aligned}
$$


where $c$ is the dimensionless contact domain $(c=\beta C)$ and here $\alpha$ is a dimensionless parameter defined as follows:

$$
\alpha=\frac{k_{2}}{k_{1}} \beta^{3 / 4}=4 \sqrt[4]{2} \sqrt{\frac{\gamma}{\pi E^{*} R}}\left(\frac{E^{*}}{2 E_{1}}\right)^{3 / 16} .
$$

Clearly here $\alpha \propto \sqrt{\gamma /\left(E^{*} R\right)}$. The adhesion effect $(\gamma)$ and cylinder size effect $(R)$ are incorporated in the parameter $\alpha$, which as shown later is a key parameter in determining the elastic deformation behavior of a micro/nanowire. This dimensionless parameter $\alpha$ also reminds us of the famous dimensionless number defined by Tabor [36] relating to contacting spheres, which is thus often referred to as Tabor number. The Tabor number holds the vital role in differentiating different contact models $[34,37,38]$. The Tabor number is defined as $\mu=\left[R_{\mathrm{s}} \gamma^{2} /\left(E^{* 2} z_{\mathrm{o}}^{3}\right)\right]^{1 / 3}$ (here $R_{\mathrm{S}}$ is the sphere radius and $z_{\mathrm{o}}$ is the equilibrium separation of atoms). The term $z_{\mathrm{o}}$ arises here because of the Lennard-Jones potential [34]. Here $\alpha$ plays the same role for a cylinder as that of $\mu$ for a sphere. One obvious distinction here is that a larger $R$ results in a smaller $\alpha$ but a larger $\mu$.

To have the finite element formulation, we introduce the following interpolation for $w$ :

$$
w=\sum_{i=1}^{4} N_{i}(\zeta) d_{i}
$$

where $\zeta=\xi / l_{\mathrm{e}} ; l_{\mathrm{e}}$ is the element length; $d_{1}$ and $d_{3}$ are the nodal displacements; $d_{2}$ and $d_{4}$ are the nodal rotations; and $N_{i}$ are the interpolation functions defined as follows [39]:

$$
\begin{aligned}
& N_{1}(\zeta)=1-3 \zeta^{2}+2 \zeta^{3}, \quad N_{2}(\zeta)=l_{\mathrm{e}} \zeta\left(1-2 \zeta+\zeta^{2}\right), \\
& N_{3}(\zeta)=\zeta^{2}(3-2 \zeta), \quad N_{4}(\zeta)=l_{\mathrm{e}} \zeta^{2}(\zeta-1) .
\end{aligned}
$$

We then substitute equation (14) into equation (12) and then use the routine finite element procedures to formulate equation (12) and a nonlinear equation set with $d_{i}$ unknowns is obtained [39]. Here keep in mind that the contact domain $c$ in equation (12) is still unknown. Physically the contact domain can only be within $\left[0, l_{1}\right] \cup\left[l_{1}+l_{\mathrm{s}}, l_{1}+l_{\mathrm{s}}+l_{2}\right]$, which are the domains where the wire is originally laid down on the trench support. Now, because the wire can lift-off/separate from the trench support during the deformation process, various contact domains such as the four typical examples shown in Fig. 1(b) can appear. Here the criterion of the lift-off is taken as a displacement constraint of $w<0$ [26, 27]. Besides the Newton-Rhapson method [40] used to solve the nonlinear equation set, we also need to check the nodal displacement one by one and once we find the lift-off points of $w<0$, the corresponding second integral term of equation (12) which accounts the energy due to contact is set to be zero. Consequently the nonlinear equation set derived from the finite element formulation is changed because of the change in the contact domains. Our solution strategy can be summarized as follows: (1) guess an 
initial contact domain (for example, the full contact case) and initial cylinder nodal displacements; (2) formulate finite element equation set according to equation (12) and the corresponding contact domain, then solve the equations set via the NewtonRhapson method; (3) check the newly solved nodal displacement to find the new contact domain; (4) go back to step (2) and iterate until the results converge. In all the finite element computations presented in this paper, the element number is fixed as 150 . The nonlinear equation set obtained by the finite element formulation is not sensitive to the initial guess of the contact domain. Usually it only takes a few iterations (less than 20) to have the solutions converge.

\section{Dimensional Analysis}

A dimensionless analysis is needed for readers to better understand our numerical results presented in the next section. Because a nondimensionalization scheme is used with equation (10), the interactions of adhesion, beam span, beam portion laid on the elastic medium and the concentrated load are incorporated in the dimensionless parameters. Although the dimensionless nature governing equation of equation (12), offers a more general study on the nanowire bending, the physical meanings of our numerical analysis can be blurred without a detailed analysis and evaluation on the those dimensionless parameters. Through this dimensional analysis, reasonings on how the parameters used in our computation are taken are also given.

Equation (11) gives the following equation:

$$
\beta=\sqrt[4]{\frac{E^{*}}{2 E_{1}}} \frac{1}{R}=D_{1} \frac{1}{R} .
$$

Here $D_{1}=\sqrt[4]{E^{*} /\left(2 E_{1}\right)}$ is a constant depending on the Young's moduli and Poisson's ratios of the two contacting materials, $E_{1}, E_{2}, v_{1}, v_{2}$, respectively. Equation (5) is derived using the JKR model [30], which is established for two similar homogeneous solids in contact [34]. Therefore, $D_{1}=\sqrt[4]{E^{*} /\left(2 E_{1}\right)} \approx 1$. The dimensionless parameter $l_{\mathrm{S}}=\beta L_{\mathrm{s}}=D_{1} L_{\mathrm{s}} / R$ of equation (10) in essence indicates the ratio of the cylinder suspension length to its radius. In the experiment by Chen $e t$ $a l$. [8, 9], $L_{\mathrm{s}}$ is fixed and $R$ is varied, which is equivalent to varying the dimensionless parameter $l_{\mathrm{s}}$ here. Large $l_{\mathrm{s}}$ physically indicates a slender beam under test and small $l_{\mathrm{s}}$ indicates a 'chunky' beam. The energy expression of equation (8) does not account for the contribution of shear, which is to say that the Euler-Bernoulli beam model is applied to the nanowires. The Euler-Bernoulli beam model requires the beam to be slender. On the other hand, $l_{\mathrm{S}}$ (or $L_{\mathrm{S}} / R$ ) should not be too large because the nanowires with large $L_{\mathrm{s}} / R$ sag $[10,11]$, which makes the above beam theory inapplicable. In the experiment of the nanowires with $L_{\mathrm{S}} / R \approx 4000$, the nanowires behave more like an elastic string rather than a beam [11]. Therefore, in all our computations $10 \leqslant l_{\mathrm{s}} \leqslant 100$. 
The term $\alpha$ in equation (12) is defined as follows by equation (13):

$$
\alpha=4 \sqrt[4]{2} \sqrt{\frac{\gamma}{\pi E^{*} R}}\left(\frac{E^{*}}{2 E_{1}}\right)^{3 / 16}=D_{2} \sqrt{\frac{\gamma}{\pi E^{*} R}} .
$$

Here $D_{2}=4 \sqrt[4]{2 / \pi^{2}}\left(E^{*} /\left(2 E_{1}\right)\right)^{3 / 16}$ is a constant depending on the material properties of two contacting bodies. As mentioned above, the dimensionless $\alpha$ parameter incorporates, together, the adhesion effect of $\gamma$ and size effect of $R$. The term $\alpha$ (also defined as $\alpha=\left(k_{2} / k_{1}\right) \beta^{3 / 4}$ by equation (13)) in essence indicates the (order of) adhesion contribution to the line load, as compared with that due to the Hertzian contact, which is directly associated with elastic foundation modulus $k_{1}$. For many different materials, $\gamma / E^{*}$ is around $10^{-9}-10^{-12} \mathrm{~m}$ [41-43]. Our computations show that in general cases, only when $\alpha \geqslant 10^{-3}$, can this dimensionless parameter have a significant impact on the deflection behavior of the wire. Physically this means that the wire radius $R$ has to be of a micron scale or smaller for $\alpha$ to be large enough to influence the wire deflection.

The dimensionless load $J$ defined in equation (10) is as follows:

$$
J=\frac{F}{4 \beta^{2} E_{1} I}=\frac{F}{\pi R^{2} \sqrt{E_{1} E^{*}}} \approx \frac{F}{E_{1} \pi R^{2}} .
$$

Here $E_{1} \pi R^{2}=E_{1} A$ (where $A=\pi R^{2}$ is the cylinder cross-section area) is the wire axial stiffness. Therefore, $J$ indicates the ratio of the vertical load to the wire axial stiffness, which should be (very) small. The maximum $J$ taken in this paper is $2 \times 10^{-2}$ and all others are between $10^{-4}$ and $10^{-3}$.

\section{Results and Discussions}

In all our results presented here, we set $L_{1}=L_{2}$ and $L_{\mathrm{s} 1}=L_{\mathrm{s} 2}=L_{\mathrm{s}} / 2$, which prescribes a symmetric configuration and loading scenario. The formulas for the deflections of the $\mathrm{H}-\mathrm{H}, \mathrm{C}-\mathrm{C}$ and $\mathrm{C}-\mathrm{H}$ beams with the beam span of $L_{\mathrm{s}}$ and the concentrated load $F$ at the center, are taken from Roark's book [12] and nondimensionalized according to equation (10) for the comparison reasons.

Figure 2 shows the cylinder beam deflections with three different $\alpha$ 's under $J=$ $5 \times 10^{-3}, l_{\mathrm{s}}=10$ and $l_{1}=2$. The dimensionless deflections of the $\mathrm{C}-\mathrm{C}$ and $\mathrm{H}-\mathrm{H}$ beams with $J=5 \times 10^{-3}$ and $l_{\mathrm{s}}=10$ are also plotted. The rotation angles of a $\mathrm{C}-\mathrm{C}$ beam at its ends $\xi=l_{1}$ and $\xi=l_{1}+l_{\mathrm{s}}$ are zero. With the symmetric load, the rotation angles of an $\mathrm{H}-\mathrm{H}$ beam at $\xi=l_{1}$ and $\xi=l_{1}+l_{\mathrm{s}}$ are with the same magnitude but the opposite sign. In Fig. 2 , the $\mathrm{H}-\mathrm{H}$ beam rotation angle at $\xi=l_{1}$ is calculated as $\theta=\arctan \left(J l_{\mathrm{s}}^{2} / 4\right)=\arctan (0.125)$ [12]. It is seen in Fig. 2 that the deflection curve of $\alpha=0$ is very similar to that of an $\mathrm{H}-\mathrm{H}$ beam. Its rotation angle at $\xi=l_{1}$ is $\arctan (0.1168)$, which is only a little smaller than that of an $\mathrm{H}-$ $\mathrm{H}$ beam. The rotation angles at $\xi=l_{1}$ of the $\alpha=4 \times 10^{-3}$ and $\alpha=1 \times 10^{-2}$ are both $\arctan (0.0275)$, which is very small and close to zero rotation angle of a $\mathrm{C}-\mathrm{C}$ beam. It also needs to point out that for beams on elastic foundation, their 


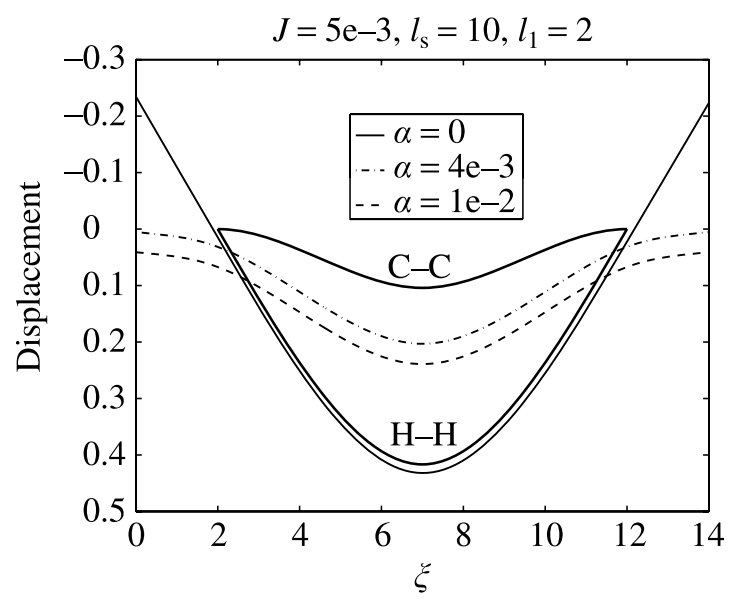

Figure 2. The beam deflections of $J=5 \times 10^{-3}, l_{\mathrm{S}}=10$ and $l_{1}=2$. The corresponding deflections of the clamped-clamped (C-C) and hinged-hinged (H-H) beams with $J=5 \times 10^{-3}$ and $l_{\mathrm{s}}=10$ are also plotted for the comparison reasons. For all the plots of the elastic foundation model presented in this paper, $\alpha$ varies as $\alpha=0,4 \times 10^{-3}$ and $1 \times 10^{-2}$.

displacements at the trench edges (i.e., $\xi=l_{1}$ and $\xi=l_{1}+l_{\mathrm{s}}$ ) are not zero. This is very obvious for the deflection curves of $\alpha=4 \times 10^{-3}$ and $\alpha=1 \times 10^{-2}$ in Fig. 2. The term $\alpha$ is also the parameter indicating the nonlinear softening effect of a spring [35]. The larger $\alpha$ here means a larger softening/adhesive effect, which requires a larger displacement to balance the same amount of exerted force [32]. The deflection curves of $\alpha=4 \times 10^{-3}$ and $\alpha=1 \times 10^{-2}$ are very similar to that of a $\mathrm{C}-\mathrm{C}$ beam. Their displacements at the trench edges are the main reason for their larger deflections than that of a $\mathrm{C}-\mathrm{C}$ beam. Now it is clear under this relatively small amount of loading $\left(J=5 \times 10^{-3}\right)$ and given geometry $\left(l_{\mathrm{s}}=10\right.$ and $\left.l_{1}=2\right)$, that adhesion does play an very important role of transforming the beam deflection from an $\mathrm{H}-\mathrm{H}$ one to a $\mathrm{C}-\mathrm{C}$ one. It is noticed that in Fig. 2 the deflection of the $\alpha=0$ curve is always larger than that of the $\mathrm{H}-\mathrm{H}$ beam. Keeping in mind that the $\mathrm{H}-\mathrm{H}$ and $\mathrm{C}-\mathrm{C}$ beams have a span of $L_{\mathrm{S}}$ and the beams of the elastic foundation model have a total span of $L_{\mathrm{s}}+L_{1}+L_{2}$. The deflection curves of $\alpha=4 \times 10^{-3}$ and $\alpha=1 \times 10^{-2}$ have the full contact scenario of case I as indicated in Fig. 1(b). There is lift-off for the $\alpha=0$ case, which has the partial contact scenario of case III. Although the elastic foundation restricts the deflection curve of $\alpha=0$ to have a slightly smaller rotation angle at $\xi=l_{1}$ than that of an $\mathrm{H}-\mathrm{H}$ beam, the facts that the longer beam is more flexible and the additional displacement at the trench edges are responsible for the larger deflection of $\alpha=0$.

In Fig. 2 we see how the adhesion changes the beam deflection under the small loading. Now let us examine how the load can change the beam deflection. In Fig. 3 the geometry of the beam is the same, with $l_{\mathrm{s}}=10$ and $l_{1}=2$ and only the load is changed to a larger one of $J=2 \times 10^{-2}$. Now we see that the deflection curves of $\alpha=0$ and $\alpha=4 \times 10^{-3}$ are almost overlapped each other. Their rotation an- 


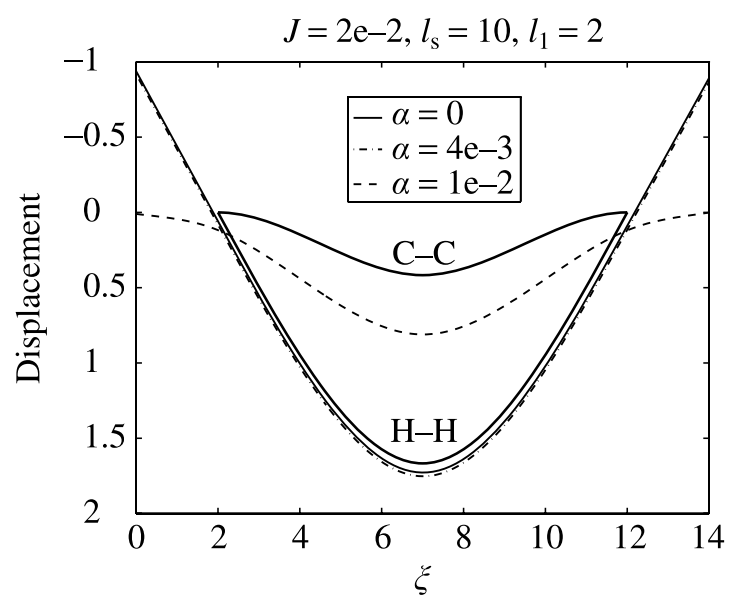

Figure 3. The beam deflections of $J=2 \times 10^{-2}, l_{\mathrm{S}}=10$ and $l_{1}=2$. The corresponding deflections of $\mathrm{H}-\mathrm{H}$ and $\mathrm{C}-\mathrm{C}$ beams are with $J=2 \times 10^{-2}$ and $l_{\mathrm{s}}=10$.

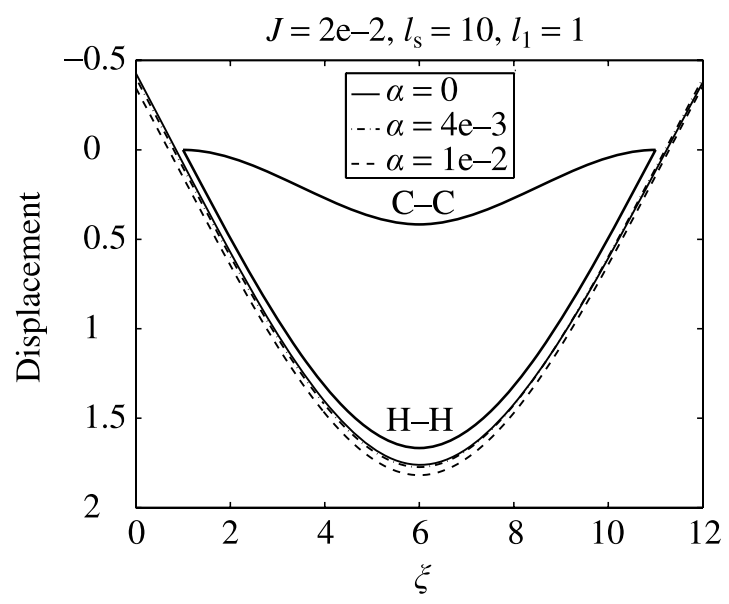

Figure 4. The beam deflections of $J=2 \times 10^{-2}, l_{\mathrm{S}}=10$ and $l_{1}=1$.

gles at $\xi=l_{1}$ are now the same as that of an $\mathrm{H}-\mathrm{H}$ beam as $\theta=\arctan \left(J l_{\mathrm{s}}^{2} / 4\right)=$ $\arctan (0.5)$. So compared with the deflection curve of $\alpha=4 \times 10^{-3}$ in Fig. 2 which is like a $\mathrm{C}-\mathrm{C}$ one, that of $\alpha=4 \times 10^{-3}$ under a larger load in Fig. 3 is now more like an $\mathrm{H}-\mathrm{H}$ one. Now both curves lift-off and have the partial contact scenario of case III. As for deflection curve of $\alpha=1 \times 10^{-2}$, it still has the full contact scenario of case I, but its rotation angle at $\xi=l_{1}$ now becomes $\arctan (0.116)$, which means the deflection curve of $\alpha=1 \times 10^{-2}$ under this larger load is neither that of a $\mathrm{C}-\mathrm{C}$ beam nor that of an $\mathrm{H}-\mathrm{H}$ beam. In Fig. 4 , we shorten $l_{1}$ as $l_{1}=1$ to see how this affects the beam deflections. In Fig. 4 all parameters are kept the same as those in Fig. 3 except for $l_{1}$. Now we see all these three deflection curves lift-off and have the contact scenario of case III. And their rotation angles at the trench 
edges are (almost) the same as that of an $\mathrm{H}-\mathrm{H}$ beam, which is $\arctan (0.5)$. The displacements due to the compression of the elastic foundation are the reason for these three deflections being larger than that of an $\mathrm{H}-\mathrm{H}$ beam. Chen et al. $[8,9]$ noticed that the boundary conditions change for a nanowire under different loads and their explanation is that under a large load the nanowire ends cannot adhere firmly to the substrate and therefore the boundary conditions become hinged ones; under a small load the nanowires adhere well to the substrate, which leads to the clamped boundary conditions. Our computation results presented in Figs 2-4 generally agree with their explanation. However, because their model does not include the adhesion and the portion length $l_{1}$ as the parameters, as demonstrated and discussed later, things are more complicated and adhesion is not the only reason.

From Figs 2-4, we see how the load, adhesion and the portion length laid down the elastic foundation affect the beam deflection behavior. In Figs $2-4$, the suspension span $l_{\mathrm{S}}$ is fixed as 10 , which physically means a relatively 'chunky' beam. Now let us examine how the slender beams behave. Compared with those in Fig. 2, $l_{\mathrm{S}}=20$ and $l_{1}=4$ in Fig. 5 are both doubled; the load $J$ is taken as $J=1 \times 10^{-3}$. Because the beam flexural rigidity is proportional to $L_{\mathrm{s}}^{3} / I=4 l_{\mathrm{s}}^{3} /(\pi R)$ [12], a much smaller load of $J=1 \times 10^{-3}$ (one fifth of that in Fig. 2) is taken in Fig. 5 to keep the small deflection in order to compare them to those of the $\mathrm{C}-\mathrm{C}$ and $\mathrm{H}-\mathrm{H}$ beams, which are derived from a linear theory. In Fig. 5 all three deflection curves with $\alpha=0,4 \times 10^{-3}, 1 \times 10^{-2}$ are between the deflection curves of a $\mathrm{C}-\mathrm{C}$ and an $\mathrm{H}-\mathrm{H}$ beam. Now for an $\mathrm{H}-\mathrm{H}$ beam the rotation angle at $\xi=l_{1}$ is $\arctan (0.1)$, the rotation angles of the three curves $\operatorname{are} \arctan \left(5.5 \times 10^{-3}\right)$ for $\alpha=0$, $\arctan \left(4.74 \times 10^{-3}\right)$ for $\alpha=4 \times 10^{-3}$ and $\arctan \left(8.95 \times 10^{-3}\right)$ for $\alpha=1 \times 10^{-2}$, respectively. The cylinder beam with $\alpha=1 \times 10^{-2}$ has the largest deflection and $\alpha=0$ has the smallest. Again, this is due to the fact that larger $\alpha$ has larger softening effect and larger displacement is required at the contact portion to balance the external force. The curves of $\alpha=0$ has the partial contact scenario of case IV and the other two have the full

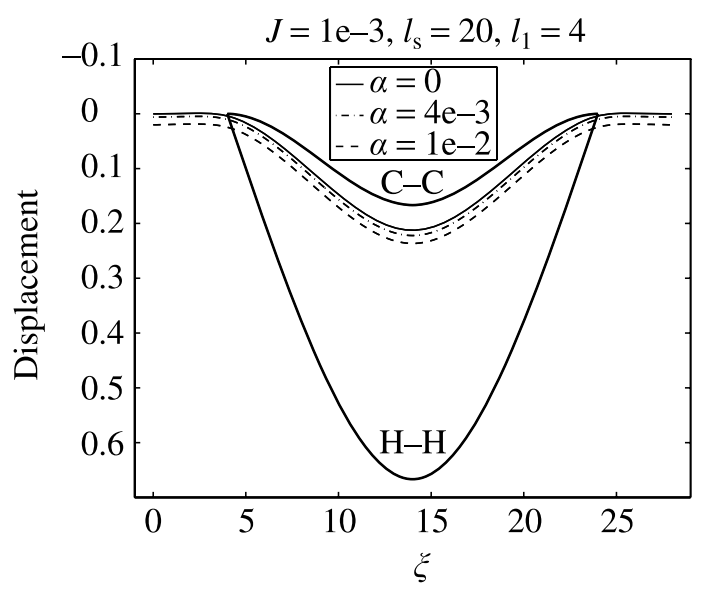

Figure 5. The beam deflections of $J=2 \times 10^{-2}, l_{\mathrm{s}}=20$ and $l_{1}=4$. 
contact scenario of case I. In Fig. 6 all the parameters are kept the same as those in Fig. 5 except that the load $J$ is changed to $J=4 \times 10^{-3}$. Similar to those in Fig. 5 the three curves in Fig. 6 are still between the two curves of a $\mathrm{C}-\mathrm{C}$ beam and an $\mathrm{H}-\mathrm{H}$ beam. Now the rotation angle of an $\mathrm{H}-\mathrm{H}$ beam becomes as $\arctan (0.4)$; the rotation angle of $\alpha=0$ is about $\arctan \left(3.3 \times 10^{-2}\right)$, the other two have the rotation of $\arctan \left(3.5 \times 10^{-2}\right)$. These three curves all have the full contact scenario of case II. In Fig. 7 we examine the effect of $l_{1}$ on the curve deflection. All the parameters in Fig. 7 are kept the same as those in Fig. 5 except that $l_{1}$ is shortened as $l_{1}=2$. The three curves are still between the curves of a $\mathrm{C}-\mathrm{C}$ beam and an $\mathrm{H}-\mathrm{H}$ beam. Now the deflection curve of $\alpha=0$ lifts-off and has the partial contact scenario of case III; its corresponding rotation angle is $\arctan \left(4.06 \times 10^{-2}\right)$. The rotation an-

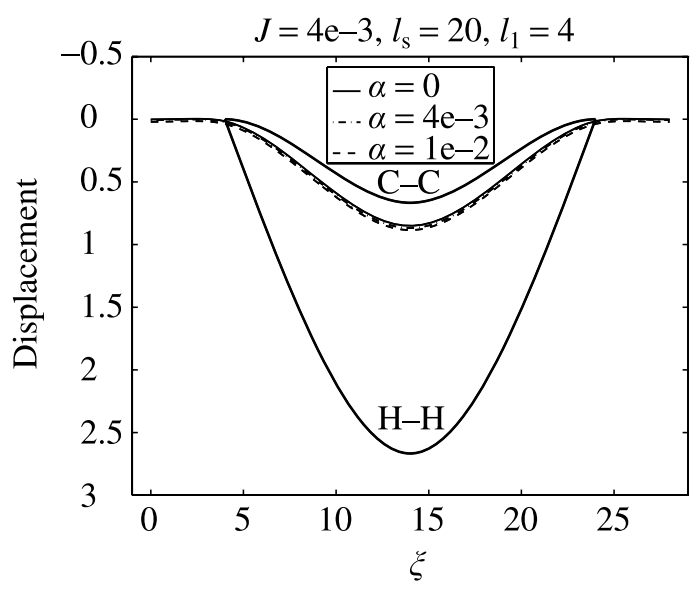

Figure 6. The beam deflections of $J=4 \times 10^{-3}, l_{\mathrm{S}}=20$ and $l_{1}=4$.

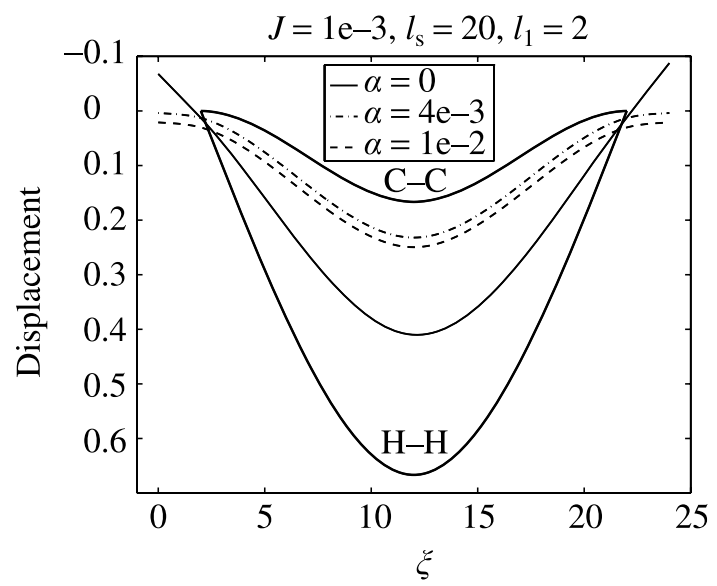

Figure 7. The beam deflections of $J=1 \times 10^{-3}, l_{\mathrm{s}}=20$ and $l_{1}=2$. 


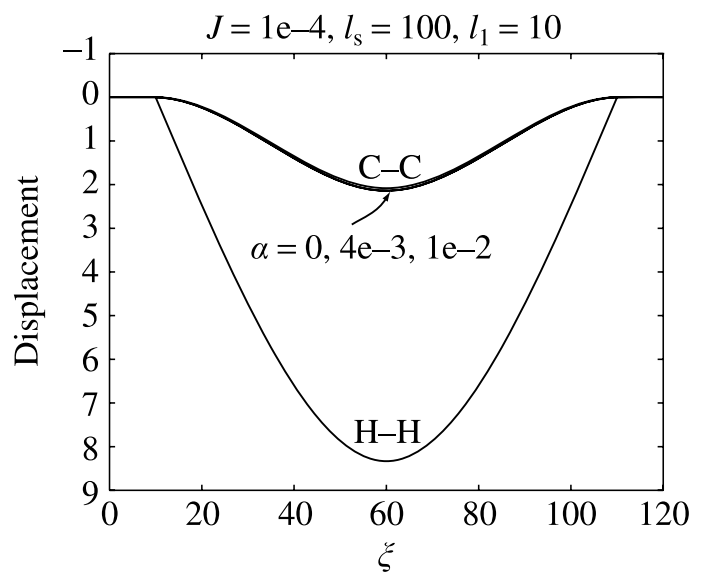

Figure 8. The beam deflections of $J=1 \times 10^{-4}, l_{\mathrm{s}}=100$ and $l_{1}=10$.

gles of $\alpha=4 \times 10^{-3}$ and $\alpha=1 \times 10^{-2}$ are both approximately $\arctan \left(3.7 \times 10^{-3}\right)$ and they both have the full contact scenario of case I.

For a very slender beam with $l_{\mathrm{s}}=100, l_{1}=10$ and $J=1 \times 10^{-4}$ as shown in Fig. 8, the three curves are almost exactly like that of a $\mathrm{C}-\mathrm{C}$ beam and it is clear that for this very slender beam its deflection is not sensitive to the adhesion. The rotation angle of $\alpha=0$ is $\arctan \left(3.1 \times 10^{-3}\right)$ and it has the partial contact scenario of case IV. The rotation angles of $4 \times 10^{-3}$ and $\alpha=1 \times 10^{-2}$ are both approximately $\arctan \left(3 \times 10^{-3}\right)$ and they also both have the full contact scenario of case II. In comparison, the rotation angle of an $\mathrm{H}-\mathrm{H}$ beam now is $\arctan (0.25)$. It also needs to emphasize here that for this very slender beam of $l_{\mathrm{s}}=100$, the beam deflection pattern of the elastic foundation model is not sensitive to both the load $J$ and the beam portion laid down on the trench $l_{1}$. In our computation we change $J$ to $J=5 \times 10^{-4}$ and $l_{1}=20$, the deflection curves look very similar to what we see in Fig. 8, that is all the curves are very close to that of $\mathrm{C}-\mathrm{C}$ beam and it is thus not plotted again.

Figure 9 shows an asymmetric deflection of the beam with $J=1 \times 10^{-4}, \alpha=$ $0, l_{\mathrm{s}}=100$ and $l_{1}=2$. The deflection of a $\mathrm{C}-\mathrm{H}$ beam is also plotted together with those of a $\mathrm{C}-\mathrm{C}$ beam and an $\mathrm{H}-\mathrm{H}$ beam. It is quite counter-intuitive that such asymmetric deflection appears in the symmetric structure $\left(l_{1}=l_{2}\right)$ under a symmetric load ( $J$ is loaded at the center). Here we need to point out the following facts: the beam model used in this paper is the Euler-Bernoulli beam theory and the load is carefully chosen in order to keep small linear deflection. There are only two sources contributing to the nonlinearity of equations (9) or (12). The first one is the adhesion, which is clearly indicated in equation (9) and is responsible for the softening effect of the elastic foundation. The second one is the contact area, which is not known a priori. This unknown property of contact area has already been pointed out as the major obstacle in solving the tensionless contact problem [22, 27]. This asymmetric deflection is reported in the experiments by Chen et al. $[8,9]$ and they 


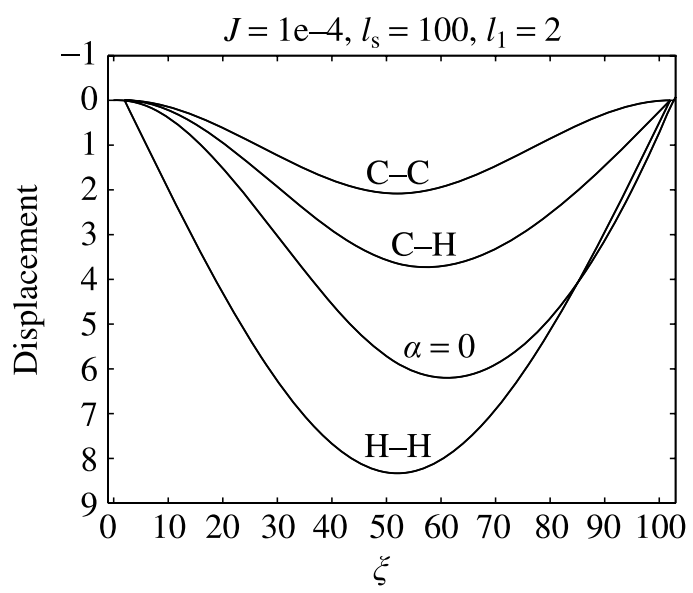

Figure 9. The beam deflections of $J=1 \times 10^{-4}, l_{\mathrm{S}}=100$ and $l_{1}=2$. The corresponding deflections of the $\mathrm{C}-\mathrm{C}, \mathrm{H}-\mathrm{H}$ and clamped-hinged $(\mathrm{C}-\mathrm{H})$ beams are also plotted for the comparison. Here the foundation model shows the asymmetric deflection.

ascribe this asymmetry vaguely to the adhesion influence on the beam boundary conditions. Because $\alpha$ is set as zero in our computation case presented in Fig. 9, physically the unknown property of the contact area (or say lift-off) is the reason for the asymmetric deflection. Mathematically, the conditions to locate the variable lift-off points are called transversality conditions [44] or matching conditions [27]. The transversality conditions together with linear governing equations form a highly nonlinear equation set $[26,27]$ and this nonlinearity due to the transversality conditions is mathematically responsible for the asymmetric deflection of the above nanowire with a symmetric configuration and loading.

Chen et al. $[8,9]$ found that for a nanowire with a large radius, the $\mathrm{H}-\mathrm{H}$ boundary conditions fit; for a nanowire with a small radius, the $\mathrm{C}-\mathrm{C}$ boundary conditions fit. In the experiments of Chen et al. [8,9], the beam suspension length $\left(L_{\mathrm{S}}\right)$ is fixed and the nanowires with different radius $R$ are tested under an AFM load at the center. As mentioned before, their large radius case corresponds to our small $l_{\mathrm{s}}$ case and their small radius case corresponds to our large $l_{\mathrm{S}}$ case. The results presented in our Figs 4 and 8 reflect the (general) observations by Chen et al. [8, 9]. In our model, $\alpha, J, l_{\mathrm{s}}$ and $l_{1}$ are studied systematically. When $l_{\mathrm{s}}$ is small, $\alpha, J$ and $l_{1}$ will all play important role of determining the beam deflection and the beam deflection curve can look like that of a $\mathrm{C}-\mathrm{C}$ beam or an $\mathrm{H}-\mathrm{H}$ beam or neither of them as indicated from Figs $2-4$. When $l_{\mathrm{S}}$ is large, the beam deflection is not sensitive to $\alpha, J$ and $l_{1}$; the deflection curves all look a $\mathrm{C}-\mathrm{C}$ one as indicated in Fig. 8. For the beam with the intermediate $l_{\mathrm{s}}$ as indicated in Figs 5-7, the beam deflection curve looks like neither a $\mathrm{C}-\mathrm{C}$ one nor an $\mathrm{H}-\mathrm{H}$ one. The contact portions in general form a constraint which makes the rotation angle between that of a $\mathrm{C}-\mathrm{C}$ beam (zero degree) and that of an $\mathrm{H}-\mathrm{H}$ beam, i.e., the intermediate boundary conditions are formed. 
Finally, we address the friction issue in the three-point bending test. Equations (9) and (12) do not consider the friction effect in the horizontal direction. When the suspended nanowire is pushed down, its mid-plane is stretched and a tension force is thus generated. This tension force is balanced by the nanowire-trench interfacial friction. When the tension reaches the critical value of static friction, the nanowire slides, which has a direct impact on the parameters of $L_{\mathrm{S}}$ and $L_{1}$. Recent experiments $[45,46]$ show that for nanowire/substrate friction, the empirical Amonton-Coulomb friction law may not be applicable and the Bowden-Tabor law should be applied. The Amonton-Coulomb friction states $F_{\text {fric }}=\mu N$ ( $F_{\text {fric }}$ : static friction; $\mu$ : coefficient of friction and $N$ : normal compressive force) and the Bowden-Tabor law states $F_{\text {fric }}=\tau A$ ( $\tau$ is the interfacial shear strength and is the $A$ is the true contact area) $[45,46]$. There are two major obstacles using the Bowden-Tabor law to model the nanowire friction in a three-point bending test: (1) because of lift-off, the nanowire shows different contact scenarios, which makes it very difficult to calculate the true contact area; (2) it is also experimentally difficult to find the interfacial strength [46]. For example, the formation of oxide layer on the nanowire surface, metallic bonding between nanowire and substrate, adhesion force induced in the fabrication process/experiment stage and even the model used to extract the interfacial shear strength can all cause the data scattering of the interfacial strength [46]. However, the interfacial shear strength is relatively large for a nanowire, for example, $\tau$ lies between 134 and $139 \mathrm{MPa}$ for $\mathrm{Ag}$ nanowire/Au substrate [46]; and the horizontal force required to cause the nanowire to slide is so large that the fracture of nanowire is observed in the experiment [45]. In general, the deflection of the suspended nanowire in a three-point bending test is small and there is little or no sliding $[4,5,7]$.

\section{Conclusion}

In this work the interactions between the nanowire geometry, adhesion and applied load are systematically studied in an adhesive receding contact model to show how they influence the nanowire deformations and its boundary conditions. Both adhesion and lift-off cause nonlinearity of the receding contact. A finite element computation is given to offer a numerical analysis. At the same time, a dimensional analysis is also presented. The following four dimensionless parameters are the control parameters used in our analysis: (1) $\alpha$, which indicates the adhesion contribution to the contact line load as compared with that of a Hertzian contact; (2) $l_{\mathrm{s}}$, which indicates the ratio of the suspension span to the cylinder radius; (3) $l_{1}, l_{2}$ ( $l_{1}=l_{2}$ are set in our study), which are the ratios of the lengths of wire portion laid on the elastic medium to the cylinder radius; (4) $J$, which is the ratio of vertical load to the cylinder axial stiffness. The asymmetric configuration $\left(l_{1} \neq l_{2}\right)$ and asymmetric loading $\left(l_{\mathrm{s} 1} \neq l_{\mathrm{s} 2}\right)$ have a definite influence on the nanowire deflections and boundary conditions. However, only the symmetric configuration and symmetric loading are studied in this paper. The above four dimensionless parameters are 
solely responsible for the results obtained from our receding contact model. The interactions and competitions between these four dimensionless parameters determine the nanowire deflection and boundary conditions. The following trends are observed: for 'chunky' nanowire with a small $l_{\mathrm{s}}$, large $\alpha$ (adhesion) and $l_{1}$ reduce lift-off, which makes the nanowire rotation difficult at the trench ends. Under a (very) small vertical load, the rotation is (very) small and the clamped-clamped boundary conditions can be a good approximation. On the other hand, larger $J$ values cause more lift-off and thus rotation increases. When $J$ is large enough, most parts lift-off and the nanowire rotates like a hinged-hinged beam. However, the general scenario is that the nanowire behaves neither like a clamped-clamped beam or a hinged-hinged beam: the nanowire rotates at the trench edges with a finite angle less than that of a hinged-hinged beam, which forms intermediate boundary conditions. The nanowire deflection of a very slender nanowire (very large $l_{\mathrm{s}}$ ) is not sensitive to $\alpha, J$ and $l_{1}$. For a very slender nanowire, the corresponding $J$ is also very small, resulting in a small deflection in the suspension part, which ensures the applicability of the Euler-Bernoulli beam theory. For a very slender nanowire under load with a $J$ value of a reasonable range, the deflections on the support are extremely small compared to that of the suspension part, and so are the rotations at the trench edges. Therefore, the nanowire behaves like a clamped-clamped beam. In summary, for a nanowire under 3-point bending test, the following competitive mechanism exists: adhesion prevents and reduces lift-off; vertical load induces and accelerates lift-off. At the same time, this competition is significantly influenced by the nanowire geometries $\left(l_{\mathrm{s}}\right.$ and $\left.l_{1}\right)$.

The boundary conditions of the nanowire in the test are key factors in interpreting the experimental data on the nanowire material properties. For the three-point bending test of the nanowire whose two ends are bonded with the supporting elastic medium by adhesion, the nanowire boundary conditions show rich patterns because of the lift-off mechanism. For the single-point/midpoint measurement of nanowire, extreme caution should be taken: adhesion may not be strong enough to hold the nanowire clamped-clamped and boundary conditions may change to intermediate one or hinged-hinged one or even a clamped-hinged one. Because the boundary condition change has a direct impact on the force-displacement measurement in the experiment, the interpretation on the nanowire material properties can lead to large differences (up to four times) in the results obtained by different researchers. Either the nanowire ends should be enhanced by an extra processing technique to make sure that they are clamped with its supporting materials or the multiple-point measurement approach should be used to monitor its changing boundary conditions.

\section{Acknowledgements}

This work is supported by the National Natural Science Foundation of China (NSFC, Grant Nos. 60936001, 11072244 and 11011120245) and National Basic Research Program of China (973 Program, Grant No. 2007CB310500). The authors 
also thank the anonymous reviewer for bringing the works on friction by Conache et al. and Zhu et al. to their attention.

\section{References}

1. Y. Zhu, C. Ke and H. D. Espinosa, Exp. Mech. 47, 7 (2007).

2. S. Cuenot, S. Demoustier-Champagne and B. Nysten, Phys. Rev. Lett. 85, 1690 (2000).

3. J. Salvetat, G. A. D. Briggs, J. Bonard, R. Bacsa, A. J. Kulik, T. Stöckli, N. A. Burnham and L. Forró, Phys. Rev. Lett. 82, 944 (1999).

4. A. Kis, S. Kasas, B. Babic, A. J. Kulik, W. Benoit, G. A. D. Briggs, C. Schönenberger, S. Catsicas and L. Forró, Phys. Rev. Lett. 89, 248101 (2002).

5. G. Jing, H. Ji, W. Yang, J. Xu and D. Yu, Appl. Phys. A 82, 475 (2006).

6. S. Cuenot, C. Frétigny, S. Demoustier-Champagne and B. Nysten, Phys. Rev. B 69, 165410 (2004).

7. G. Jing, H. Duan, X. Sun, Z. Zhang, J. Xu, Y. Li, J. Wang and D. Yu, Phys. Rev. B 73, 235409 (2006).

8. Y. Chen, B. L. Dorgan, D. N. Mcllroy and D. E. Aston, J. Appl. Phys. 100, 104301 (2006).

9. Y. Chen, I. Stevenson, R. Pouy, L. Wang, D. N. Mcllroy, T. Pounds, M. G. Norton and D. E. Aston, Nanotechnology 18, 135708 (2007).

10. B. Wu, A. Heidelberg, J. J. Boland, J. E. Sader, X. Sun and Y. Li, Nano Letters 6, 468 (2006).

11. D. A. Walters, L. M. Ericson, M. J. Casavant, J. Liu, D. T. Colbert, K. A. Smith and R. E. Smalley, Appl. Phys. Lett. 31, 3803 (1999).

12. R. J. Roark, Formulas for Stress and Strain, Chapter 8, 3rd edn. McGrall-Hill Book Company, New York, NY (1954).

13. S. Hoffman, I. Utke, B. Moser, J. Michler, S. H. Christiansen, V. Schmidt, S. Senz, P. Werner, U. Gösele and C. Ballif, Nano Letters 6, 622 (2006).

14. Y. Zhang, Q. Ren and Y. Zhao, J. Phys. D: Appl. Phys. 37, 2140 (2004).

15. Y. Zhang and Y. Zhao, J. Appl. Phys. 99, 053513 (2006).

16. H. S. Park and P. A. Klein, J. Mech. Phys. Solids 56, 3144 (2008).

17. W. Fang and J. A. Wickert, J. Micromech. Microeng. 6, 301 (1996).

18. Y. Zhang and Y. Zhao, Microsyst. Technol. 12, 357 (2006).

19. A. San Paulo, J. Bokor, R. T. Howe, R. He, P. Yang, D. Gao, C. Carraro and R. Maboudian, Appl. Phys. Lett. 87, 053111 (2005).

20. L. M. Keer, J. Dundurs and K. C. Tsai, J. Appl. Mech. 39, 1115 (1972).

21. M. Ratwani and F. Erdogan, Int. J. Solids Structures 9, 921 (1973).

22. Y. Weitsman, J. Appl. Mech. 36, 198 (1969).

23. S. L. Pu and M. A. Hussain, J. Appl. Mech. 37, 859 (1970).

24. G. M. Gladwell, J. Appl. Mech. 43, 263 (1976).

25. Y. Weitsman, Int. J. Engng. Sci. 10, 73 (1972).

26. Y. Weitsman, J. Appl. Mech. 37, 1019 (1970).

27. Y. Zhang and K. D. Murphy, Int. J. Solids Struct. 41, 6745 (2004).

28. M. Yu, T. Kowalewski and R. S. Ruoff, Phys. Rev. Lett. 85, 1456 (2000).

29. G. Feng, W. Nix, Y. Yoon and C. Lee, J. Appl. Phys. 99, 074304 (2006).

30. M. K. Chaudhury, T. Weaver, C. Y. Hui and E. J. Kramer, J. Appl. Phys. 80, 30 (1996).

31. S. Chen and H. Gao, Proc. R. Soc. A 462, 211 (2006).

32. K. L. Johnson, Contact Mechanics, Chapters 4 and 5. Cambridge University Press, Cambridge (1985).

33. A. D. Kerr, J. Appl. Mech. 31, 491 (1964). 
34. Y. Zhang, J. Adhes. Sci. Tech. 22, 699 (2008).

35. A. H. Nayfeh and D. T. Mook, Nonlinear Oscillations, Chapter 2. Wiley, New York, NY (1979).

36. D. Tabor, J. Colloid Interface Sci. 58, 2 (1977).

37. Y. Zhao, L. S. Wang and T. X. Yu, J. Adhes. Sci. Tech. 17, 519 (2003).

38. K. L. Johnson and J. A. Greenwood, J. Colloid Interface Sci. 192, 326 (1997).

39. K. J. Bathe, Finite Element Procedures, Chapters 5 and 6. Prentice-Hall, Inc., New Jersey (1996).

40. W. H. Press, S. A. Teukolsky, W. T. Vetterling and B. P. Flannery, Numerical Recipes in Fortran, 2nd edn, Chapter 9. Cambridge University Press (1992).

41. R. E. Miller and V. B. Shenoy, Nanotechnology 11, 139 (2000).

42. M. Giri, D. B. Bousfield and W. N. Unertl, Langmuir 17, 2973 (2001).

43. Y. Sun, B. Akhremitchev and G. C. Walker, Langmuir 20, 5837 (2004).

44. A. D. Kerr, Int. J. Solids Structures 12, 1 (1976).

45. G. Conache, S. M. Gray, A. Ribayrol, L. E. Fröberg, L. Samuelson, H. Pettersson and L. Montelius, Small 5, 203 (2009).

46. Y. Zhu, Q. Qin, Y. Gu and Z. L. Wang, Nanoscale Res. Lett. 5, 291 (2010). 